\title{
Salidroside-pretreated mesenchymal stem cells contribute to neuroprotection in cerebral ischemic injury in vitro and in vivo
}

\section{Liping Zhou}

Zhejiang Chinese Medical University

\section{Panpan Yao}

Zhejiang Chinese Medical University

\section{Lixia Jiang}

Zhejiang Chinese Medical University

\section{Zhaoyun Wang}

Zhejiang Chinese Medical University

\section{Xiaohe Ma}

Zhejiang Chinese Medical University

\section{Guangxin Wen}

Zhejiang Chinese Medical University

\section{Jintao Yang}

Zhejiang Chinese Medical University

\section{Binjie Zhou}

Zhejiang Chinese Medical University

Qin Yu ( $\sim$ qinyu3587@126.com )

Zhejiang Chinese Medical University https://orcid.org/0000-0002-8355-2438

\section{Research}

Keywords: Mesenchymal stem cells, Salidroside, Cerebral ischemic injury, Cell survival, Neuroprotection

Posted Date: August 18th, 2020

DOI: https://doi.org/10.21203/rs.3.rs-59580/v1

License: (c) (1) This work is licensed under a Creative Commons Attribution 4.0 International License. Read Full License

Version of Record: A version of this preprint was published at Journal of Molecular Histology on September 27th, 2021. See the published version at https://doi.org/10.1007/s10735-021-10022-0. 


\section{Abstract}

Background: Mesenchymal stem cells (MSCs) are considered a promising tool for treating cerebral ischemic injury. However, their poor survival after transplantation limits their therapeutic effect and applications. Salidroside has been reported to exert potent cytoprotective and neuroprotective effects. This study aimed to investigate whether salidroside could improve MSC survival under hypoxic-ischemic conditions and, subsequently, alleviate cerebral ischemic injury in a rat model.

Methods: MSCs were pretreated by salidroside under hypoxic-ischemic conditions. The cell proliferation, migratory capacity, and apoptosis were evaluated by means of Cell Counting Kit (CCK)-8, transwell assay, and flow cytometry. MSCs pretreated with salidroside were transplanted into the rats subsequent to middle cerebral artery occlusion. The grip strength, 2,3,5-triphenyltetrazolium chloride, and hematoxylineosin staining were used to analyze the therapeutic efficiency and pathological changes. The mature neuron marker NeuN and astrocyte marker GFAP in the focal area were detected by immunofluorescence.

Results: These results indicated that salidroside promoted the proliferation, migration and reduced apoptosis of MSCs under hypoxic-ischemic conditions. In vivo experiments revealed that transplantation of salidroside-pretreated MSCs strengthened the therapeutic efficiency by enhancing neurogenesis and inhibiting neuroinflammation in the hippocampal CA1 area after ischemia.

Conclusion: Our results suggest that pretreatment with salidroside could be an effective strategy to enhance the cell survival rate and the therapeutic effect of MSCs in treating cerebral ischemic injury.

\section{Background}

Brain hypoxia-ischemia has been considered a critical factor in many human central nervous system diseases, including stroke, which is the leading cause of disability and the second most common cause of mortality worldwide [1, 2]. Although progress has been made on fundamental research and clinical treatment, there are no efficient therapies to ameliorate the resulting neurodegeneration.

Mesenchymal stem cells (MSCs) have displayed capabilities in regenerative therapy for the treatment of stroke because of their multi-directional differentiation potential and hypo-immunogenicity properties [3]. Transplantation of MSCs enhances functional recovery and endogenous neurogenesis in stroke models $[4,5]$. The cell survival rate after MSCs are transplanted into the ischemic region is quite low, which has limited the application of MSCs [6-8]. Hence, promoting cell survival and migration potential of the MSCs is one of the critical issues for stem cell-based therapy for cerebral ischemic injury.

Salidroside (p-hydroxyphenethyl- $\beta$-D-glucoside, Sal), aphenylpropanoid glycoside isolated from the Rhodiola rosea, has been widely used as a traditional herbal medicine that exhibits potent antiinflammatory and anti-apoptotic functions [9]. Previous studies have shown that salidroside could promote the proliferation of MSCs [10] and induce MSCs to differentiate into cholinergic neurons [11] and 
dopaminergic neurons [12]. Concurrently, salidroside has been reported to improve brain ischemic injury by reducing neuroinflammation and neural damage [13-15].

In the present study, we therefore investigated the effect of salidroside on survival and migration potential of MSCs under hypoxia-ischemia and focused on the therapeutic effect by which salidroside-pretreated MSCs induce neuroprotective effects following cerebral ischemic injury.

\section{Methods}

\section{Animals}

In the present study, male Sprague-Dawley (SD) rats (80-120 g) were used for MSC isolation and culture. SD rats (260-300 g) were used for middle cerebral artery occlusion (MCAO) model establishment. All rats were provided by Zhejiang Chinese Medical University Animal Center (Laboratory Animal Certificate: SYXK 2013-0184). Rats were raised in individual cages in a temperature- and humidity-controlled room with a 12-h light/dark cycle and access to food and water ad libitum.

\section{MSC culture and pretreatment}

MSCs were isolated from SD rats (80-120 g) under sterile environment as previously reported [16]. The long bone was taken from the hind legs of SD rats, and both ends of the long bones were cut. Bone marrow was flushed out with phosphate buffered saline (PBS, BOSTER, China). The obtained bone marrow solution was centrifuged at 1,000 rpm for $5 \mathrm{~min}$, the supernatant liquid was removed, and the cell pellets were resuspended in Dulbecco's Modified Eagle's Medium/Ham's F-12 (DMEM/F12, Gibco, USA) supplemented with $10 \%$ fetal bovine serum (FBS, Gibco, USA) and 1\% penicillin-streptomycin (Gibco, USA). Cells were detached with $0.25 \%$ trypsin-ethylene diamine tetraacetic acid (EDTA, Gibco, USA) at $80 \%$ confluence. Passage 3 cells were used for the following experiments.

For hypoxia and ischemia, MSCs were pretreated with serum-free medium with $100 \mu \mathrm{mol} / \mathrm{l}$ of Cobalt chloride ( $\mathrm{CoCl}_{2}$, Sigma, USA) for $12 \mathrm{~h}$. For salidroside (HPLC $\geq 98 \%$, Shanghai Yuanye Bio-Technology Co., Ltd., China) pretreatment, MSCs were cultured with different concentrations of salidroside $(0.375,0.75$, $1.5,3,6 \mu \mathrm{g} / \mathrm{ml}$ ) for $48 \mathrm{~h}$, followed by exposure to $100 \mu \mathrm{mol} / \mathrm{l}$ of $\mathrm{CoCl}_{2}$ with serum-free medium for $12 \mathrm{~h}$. The MSCs cultured in complete medium served as control. These pretreated cells were used for Cell Counting Kit (CCK)-8 assay, transwell assay, and flow cytometry assay.

CM-Dil (Invitrogen, Carlsbad, CA, USA) was used to label the cell membrane prior to transplantation, without affecting cell morphology, viability, and proliferation capacity. CM-Dil stock solution was prepared as recommended by the manufacturer.

\section{CCK-8 assay}

MSC proliferation was measured using the CCK-8 (Dojindo Laboratories, Inc., Kumamoto, Japan). MSCs were inoculated at $1 \times 10^{3}$ cells/well in 96 -well culture plates. The medium was discarded, and each well 
was washed twice with PBS. One hundred microliters of DMEM/F12 and $10 \mu \mathrm{l}$ of CCK-8 reagents were added to each well and incubated at $37^{\circ} \mathrm{C}$ with $5 \% \mathrm{CO}_{2}$ for $2 \mathrm{~h}$. The absorbance of the reaction solution was measured at $450 \mathrm{~nm}$ using a microplate reader (SpectraMax Plus384, Molecular Devices, LLC, Sunnyvale, CA, USA).

\section{Transwell assay}

Cell migration ability was performed using transwell plates (24-well plate, 8- $\mu$ m pore size, Corning, NY, USA). Cells were digested and resuspended in DMEM/F12 containing $2 \%$ FBS. One hundred fifty microliters $\left(1 \times 10^{5}\right.$ cells $\left./ \mathrm{ml}\right)$ of cell suspension was added to the upper chamber of the migration well, and $600 \mu \mathrm{l}$ of complete medium was added to the lower chamber. After $15 \mathrm{~h}$, cells from the top of the filter were carefully removed with a cotton swab. The cells that had migrated through to the underside of the insert membranes were then fixed with $4 \%$ paraformaldehyde for 30 min and stained with crystal violet for $10 \mathrm{~min}$. Migrated cells were observed and imaged by a fluorescence microscope (Nikon, Eclipse Ts2-FL, Japan). The number of migrated cells was determined by averaging 6 random fields per well.

\section{Flow cytometry assay}

To quantify the apoptotic MSCs, cells were collected, washed with PBS, and then centrifuged at 1,000 rpm for 5 min before $1 \times 10^{5}$ cells were resuspended in the $1 \times$ AnnexinV binding buffer mixed with $5 \mu$ of propidium iodide $(\mathrm{PI})$ reagent. After incubation for $15 \mathrm{~min}$ at room temperature in the dark, another $400 \mu \mathrm{l}$ of binding buffer was added, and cells were measured using a flow cytometry machine (Accuri C6, BD Biosciences)

\section{MCAO model}

MCAO was induced by the modified Zea Longa method [17]. Rats were anesthetized with $10 \%$ chloral hydrate $(0.35 \mathrm{ml} / 100 \mathrm{~g}$, Yonghua Chemical, China) and fixed on an operating table in a supine position. A midline neck incision was made, and the common carotid artery (CCA) and its bifurcation were exposed and isolated carefully from the vagus nerve. The bifurcation of the external common carotid artery and the proximal end of CCA were occluded by a silk suture. A small hole was cut into the CCA to insert a filament into the internal CCA. The suture was tightly tied around the filament to prevent bleeding and the reverse-action tweezers were removed. After $1.5 \mathrm{~h}$ of occlusion, the suture was withdrawn to enable reperfusion and the wound and skin were sutured. The rats in the sham group received the same procedures, except that the MCAO group was not occluded. Finally, animals were placed in a cage over a warming blanket until full recovery from anesthesia.

\section{Transplantation of MSCs and animal grouping}

Rats were randomly divided into 4 groups, including the sham, vehicle, MSCs (MSCs transplantation), and Sal-MSCs (cells pretreated with $0.75 \mu \mathrm{g} / \mathrm{ml}$ salidroside). Three days after MCAO, the anesthetized rats were fixed on a stereotaxic brain locator. For the MSC and Sal-MSC groups, $10 \mu$ l of cells $\left(1 \times 10^{7}\right.$ 
cells $/ \mathrm{ml}$ ) were engrafted at $3.6 \mathrm{~mm}$ toward the caudal side of the frontal fontanel and at $2 \mathrm{~mm}$ on the right side of the midline. The depth of the injection was $3 \mathrm{~mm}$, and the rate of the injection was $1 \mu \mathrm{l} / \mathrm{min}$. Rats of the vehicle group were transplanted with the same volume of PBS. The rats were euthanized 14 days after MCAO to obtain the brain tissue samples for 2,3,5-triphenyltetrazolium chloride (TTC) staining ( $n=3$ per group), hematoxylin-eosin (HE) staining ( $n=3$ per group), and immunofluorescence ( $n=6$ per group). Behavioral testing (each time point; $n=3$ per group) was performed at 7,14 , and 21 days after MCAO.

\section{Behavioral Test}

Grip strength was measured at 7, 14, and 21 days after MCAO as previously described [18]. Briefly, each rat was held by its tail and gripped the tension bar of the instrument using their limbs. Once the grip was secured, the animal was slowly pulled away from the bar. A digital reading (in Newtons) of 3 successive trials was obtained for each rat, and then averaged for analysis.

\section{TTC staining}

To measure infarct volumes, rats were decapitated at 14 days after MCAO. Brains were cut into 2-mmthick coronal sections and incubated in $2 \%$ TTC (Sigma, USA) solution for $30 \mathrm{~min}$ at $37^{\circ} \mathrm{C}$ in the dark. Infarct volumes were determined by the ImagePro Plus 6.0 software and expressed as the percentage of the total volume of the cerebral tissue.

\section{Preparation of paraffin section}

Brains from anesthetized rats were fixed by transcardial perfusion with saline, followed by perfusion and immersion in $4 \%$ paraformaldehyde. Then, brains were removed, post-fixed, dehydrated in graded alcohol, and embedded in paraffin. Coronal sections at $4 \mu \mathrm{m}$ were used for HE staining and immunofluorescence.

\section{HE staining}

Paraffin sections were subjected to conventional dewaxing, hydration, hematoxylin staining, and hydrochloric acid ethanol differentiation. After rinsing with distilled water, the sections were stained with $1 \%$ eosin solution (BOSTER, China) for 1 min with subsequent dehydration in graded alcohol; the sections were dewaxed in xylene and mounted with neutral gum. The mounted slides were observed and imaged by microscope.

\section{Immunofluorescence}

CM-Dil-labeled MSCs were observed by fluorescence microscopy to detect the implanted cells in the host brain. Neuronal nuclear antigen (NeuN) and glial fibrillary acidic protein (GFAP) expression were assessed by evaluating the number of NeuN- and GFAP-positive cells in the ischemic boundary zone. Sections were dewaxed, hydrated, repaired by citric acid antigen, and incubated with $3 \% \mathrm{H}_{2} \mathrm{O}_{2}$ for 10 min at room temperature. Then, sections were incubated with rabbit anti-NeuN antibody $(1: 5,000, A b c a m)$ and rabbit 
anti-GFAP antibody $\left(1: 5,000\right.$, Abcam) overnight at $4^{\circ} \mathrm{C}$ and incubated with a secondary antibody at $37^{\circ} \mathrm{C}$ for 30 min. Whole sections were counter-stained with 4',6-diamidino-2-phenylindole (DAPI, Sigma, USA). Ten fields of each section were randomly imaged at high magnification (400x) to count the CM-Dillabeled MSCs NeuN- and GFAP-positive cells. Positive cells were counted by ImagePro Plus 6.0.

\section{Statistical analysis}

All experimental data are expressed as the mean \pm standard deviation. The differences between multiple groups were analyzed by one-way analysis of variance (ANOVA). Comparisons between two groups were performed via the Dunnett's t-test. All were considered statistically significant for $P<0.05$.

\section{Results}

\section{Salidroside promoted MSC proliferation}

MSCs were cultured and passaged until passage 3 . The cell morphology tends to be uniform, showing a spindle shape with high uniformity. To determine the influence of salidroside on MSC proliferation, the number of MSCs was measured using the CCK-8 test. As shown in Fig. 1a, the proliferation rate of MSCs within hypoxic-ischemic treatment was significantly reduced. In addition, there was an increase in the MSCs treated by different concentrations of salidroside $(0.375,0.75,1.5,3,6 \mu \mathrm{g} / \mathrm{ml})$ (Fig. 1a). These results indicated that salidroside promotes cell proliferation under hypoxic-ischemic conditions.

\section{Salidroside improved MSC migration}

Transwell assay was performed to determine the migration potential of MSCs, and the migrated cells were detected (Fig.1b). Compared to normoxic conditions, the migration of MSCs are suppressed under hypoxic-ischemic conditions. Concomitantly, we found that MSCs migrated to the lower compartment more rapidly in response to the salidroside $(0.375,0.75,1.5,3,6 \mu \mathrm{g} / \mathrm{ml})$, especially in the $0.75 \mu \mathrm{g} / \mathrm{ml}$ of salidroside treatment (Fig. 1c). These results confirmed that salidroside promoted the migration of MSCs against hypoxia-ischemia.

\section{Salidroside protected MSCs from apoptosis}

We analyzed the effect of salidroside pretreatment on the apoptosis rate of MSCs. Data revealed that exposure of MSCs to hypoxic-ischemic conditions induced MSCs apoptosis, whereas salidroside decreased the percentage of apoptotic cells, especially in the $0.75 \mu \mathrm{g} / \mathrm{ml}$ of salidroside treatment (Fig. 2). Such findings suggested that the number of MSCs which were apoptotic upon treatment with hypoxiaischemia were restored by salidroside pretreatment.

\section{Salidroside-pretreated MSCs ameliorated brain injuries in MCAO rats}

To evaluate the therapeutic effects of Sal-MSCs, the cells were labeled with CM-Dil, a fluorescence marker in vivo (Fig. 3), and then $1 \times 10^{5} \mathrm{MSC}$ or Sal-MSCs were transplanted into the ischemic boundary zone 3 
days after MCAO. The grip strength was investigated in the sham, vehicle, and Sal-MSC groups, respectively, on days 7,14 , and 21 . Deficits in grip strength reached their lowest point in ischemic injury rats, whereas the grip force of Sal-MSCs group was significantly elevated compared to the rats in MSC group (Fig. 4a). We compared the infarction areas in coronal sections from animals of the vehicle, MSC, and Sal-MSC groups on day 14 (Fig. 4b). TTC staining was used to assess lesion volume as a percentage of contralateral hemispheric volume. Fourteen days after MCAO, significant differences of reduction in infarct volume were detected in the Sal-MSC and MSC groups compared with the vehicle group (Fig. 4c).

\section{Salidroside-pretreated MSCs alleviated pathological changes in the hippocampal CA1 area after ischemia}

After transplantation of MSCs was accepted, CM-Dil labeled MSCs were found in the hippocampal CA1 area of rat brains (Fig. 5a). HE staining provided an objective assessment on pathological changes (Fig. 5b). Examinations of the hippocampus showed that the morphological structures of the hippocampus in the sham group were normal, with uniform dyeing. By contrast, data showed that degeneration and necrosis of nerve cells in hippocampal formations were aggravated in rats that suffered from ischemia. Similar but slight pathological changes were observed in the MSC group subsequent to ischemia; however, the changes in the MSCs with salidroside pretreatment were less marked than those of the vehicle group. This observation suggested that the transplantation of MSCs ameliorated pathological changes after cerebral ischemic injury, while salidroside pretreatment could strengthen therapeutic efficiency.

\section{Salidroside-pretreated MSCs enhanced neurogenesis in the ischemic hippocampus}

To investigate whether these transplanted MSCs could differentiate into mature neurons, CM-Dil labeling of the cells with the mature neuron marker NeuN was performed. Fig. 6a shows that many neurons were positive in the hippocampal CA1 area. However, only a few CM-Dil-positive cells co-labeled with NeuN were observed in the MSCs group. As expected, there were more double-labeled cells in the Sal-MSCs group (Fig. 6b). These data provided evidence that transplantation of Sal-MSCs enhanced neurogenesis to a greater extent than transplantation of MSCs alone.

In addition, cells with astrocyte marker GFAP were counted in the same area (Fig. 7a). The GFAP-positive cell number in the vehicle group was significantly increased in comparison with the sham group, suggesting that astrogenesis was stimulated by ischemic injury. A remarkable decrease of GFAP-positive cells was counted in both MSC-transplanted groups and the vehicle group (Fig. 7b). Furthermore, statistical analysis showed more reduction in the Sal-MSC group, which suggested that MSCs pretreated by salidroside inhibited neuroinflammation in the ischemic hippocampus.

\section{Discussion}

Stem cell therapy based on MSCs has emerged as a promising strategy for treating neurological diseases [19]. However, previous studies have shown that the therapeutic effect of MSCs is limited due to their poor survival after transplantation [20,21]. Thus, improving the survival rate of MSCs is crucial for 
achieving a successful MSC-based therapy for cerebral ischemic injury. In the present study, we first found that salidroside had a significant effect on MSC proliferation, migration, and survival and reversed the injury induced by hypoxia-ischemia in vitro. Then, MSC pretreatment with salidroside revealed the reduced infarct rate and ameliorated pathological changes after transplantation. Furthermore, the results also demonstrated the elevated number of NeuN-positive cells and decreased number of GFAP-positive cells in the ischemic hippocampus. Taken together, our findings clearly indicated that salidroside pretreatment has neuroprotective effects, which prompted further investigation of the underlying mechanisms.

Hypoxia-ischemia-induced neuroinflammation typically causes neurological damage and can occur during stroke, neonatal hypoxic-ischemic encephalopathy, and other diseases [22]. $\mathrm{CoCl}_{2}$ is a chemical compound, which is widely acknowledged as a classical stimulator to hypoxic-ischemic conditions. Here, we used serum-free medium with $100 \mu \mathrm{mol} / \mathrm{I} \mathrm{CoCl}_{2}$ for hypoxia-ischemia induction. The proliferation, migration, and survival rate of MSCs were suppressed compared to normal conditions. Qin et al.[23] reported that Rhodiola rosea could promote the proliferation and differentiation of neural stem cells in the hippocampus of the depressive rats and play a role in saving injured neurons of the hippocampus. Salidroside is a main component of Rhodiola rosea, which has been widely used as medicine in Asia and Europe and possesses various medicinal properties, such as anti-inflammatory [24], antioxidative [25], and neuroprotective effects [26-28]. Studies have shown that salidroside can inhibit the apoptosis of many normal cells $[29,30]$. In addition, salidroside could alleviate hypoxia-induced viability impairment and apoptosis in neural stem cells and pulmonary arterial smooth muscle cells [31, 32]. Our results prompt the possibility that salidroside might be able to promote the survival and migration potential of MSCs and inhibit its apoptosis.

Studies have suggested that MSCs could control inflammation by reconstructing the neural microenvironment [33]. MSCs can secrete a series of growth factors, cytokines, chemokines, and various enzymes, playing an important role in cell growth and migration [34]. A report from Yun et al. [35] has demonstrated that MSCs significantly inhibited the release of inflammatory cytokines, alleviated cognitive impairment, and increased the survival rate of neurons and nerve regeneration. Furthermore, $\mathrm{Ha}$ et al. [36] confirmed that salidroside could promote the survival of MSCs and combined to repair damaged neurons within spinal cord tissue. Our results showed that Sal-MSCs contributed to a significant decrease in the infarct rate and greatly enhanced neurogenesis in the focal area of MCAO rats. Several sudies have revealed that salidroside could reduce cerebral ischemic injury [13, 37, 38]. According to previous studies, the underlying mechanisms of salidroside against injury are due to inhibition of apoptosis and suppression of inflammation with good efficiency and low toxicity [15]. More importantly, the combination with MSCs may enhance the efficacy of salidroside. As shown in our in vivo results, the transplantation of Sal-MSCs strengthened the therapeutic efficiency by enhancing neurogenesis and inhibiting neuroinflammation in the hippocampus. Our results showed an increased number of NeuNpositive cells and a decreased number of GFAP-positive cells in the Sal-MSC group, which demonstrated that the transplantation of Sal-MSCs could promote the differentiation of neurons in the lesions and 
alleviate inflammation caused by brain injury. Although further investigation is needed for confirmation before clinical application, our results showed that MSCs possessed curative effect to some extent, while salidroside-treated MSCs exert even better efficacy.

\section{Conclusion}

Our results clearly demonstrated that salidroside could promote the proliferation, migration, and reduced apoptosis of MSCs under hypoxic-ischemic conditions. Further in vivo experiments revealed that transplantation of Sal-MSCs strengthened the therapeutic efficiency by enhancing neurogenesis and inhibiting neuroinflammation in the hippocampal CA1 area after ischemia. These findings will open up the possibility of using this combination therapy for other MSC-based treatments.

\section{Abbreviations}

CCA: common carotid artery; CCK-8: cell counting kit-8; DAPI: 4',6-diamidino-2-phenylindole; FBS: fetal bovine serum; GFAP: glial fibrillary acidic protein; HE: hematoxylin-eosin; MCAO: middle cerebral artery occlusion; MSCs: mesenchymal stem cells; NeuN: neuronal nuclear antigen; PBS: phosphate-buffered saline; TTC: 2,3,5-triphenyltetrazolium chloride.

\section{Declarations}

\section{Ethics approval and consent to participate}

Not applicable.

\section{Consent for publication}

Not applicable.

\section{Availability of data and materials}

The datasets used and/or analyzed during the current study are available from the corresponding author on reasonable request.

\section{Competing interests}

The authors declare that they have no competing interests.

\section{Funding}

This work was financially supported by the National Natural Science Foundation of China (31570994); Project of Zhejiang Education Department (Y20163679).

\section{Authors' contributions}


QY conceived the idea and designed the experiments. LPZ and PPY performed the experiments and wrote the manuscript. LXJ performed the animal experiments. ZYW and XHM performed the cellular experiment. GXW and JTY participated in part of the animal experiments. BJZ collected and analyzed the data. All authors read and approved the final manuscript.

\section{Acknowledgments}

Not applicable.

\section{References}

1. Chen JX, Liu J, Hu F, Bi Y, Li M, Zhao L. Genetic variants on chromosome 9p21 confer risks of cerebral infarction in the Chinese population: a meta-analysis. Int J Immunopathol Pharmacol. 2019;33:2058738419847852.

2. Mangin G, Poittevin M, Charriaut-Marlangue C, Giannesini C, Merkoulova-Rainon T, Kubis N. Glatiramer acetate reduces infarct volume in diabetic mice with cerebral ischemia and prevents longterm memory loss. Brain Behav Immun. 2019;80:315-27.

3. Zomer HD, Vidane AS, Gonçalves NN, Ambrósio CE. Mesenchymal and induced pluripotent stem cells: general insights and clinical perspectives. Stem Cells Cloning. 2015;8:125-34.

4. Zhang Y, Chopp M, Meng Y, Katakowski M, Xin H, Mahmood A, et al. Effect of exosomes derived from multipluripotent mesenchymal stromal cells on functional recovery and neurovascular plasticity in rats after traumatic brain injury. J Neurosurg. 2015;122(4):856-67.

5. Cunningham CJ, Redondo-Castro E, Allan SM. The therapeutic potential of the mesenchymal stem cell secretome in ischaemic stroke. J Cereb Blood Flow Metab. 2018;38(8):1276-92.

6. Zhang K, Zhao X, Chen X, Wei Y, Du W, Wang Y, et al. Enhanced Therapeutic Effects of Mesenchymal Stem Cell-Derived Exosomes with an Injectable Hydrogel for Hindlimb Ischemia Treatment. ACS Appl Mater Interfaces. 2018;10(36):30081-91.

7. Feng G, Zhang J, Li Y, Nie Y, Zhu D, Wang R, et al. IGF-1 C Domain-Modified Hydrogel Enhances Cell Therapy for AKI. J Am Soc Nephrol. 2016;27(8):2357-69.

8. Hedhli J, Konopka CJ, Schuh S, Bouvin H, Cole JA, Huntsman HD, et al. Multimodal Assessment of Mesenchymal Stem Cell Therapy for Diabetic Vascular Complications. Theranostics. 2017;7(16):3876-88.

9. Bai H, Wang CB, Ma XH, Wei YP, Xi R, Zhao Q, et al. [Effects of salidroside on proliferation of bone marrow mesenchymal stem cells]. Zhongguo shi yan xue ye xue za zhi. 2014;22(4):1072-7.

10. Zhao HB, Qi SN, Dong JZ, Ha XQ, Li XY, Zhang QW, et al. Salidroside induces neuronal differentiation of mouse mesenchymal stem cells through Notch and BMP signaling pathways. Food Chem Toxicol. 2014;71:60-7.

11. Zhang M, Zhao H, Li Z, Yang Y, Wen Y, Dong J, et al. [Effect of salidroside on rat bone marrow mesenchymal stem cells differentiation into cholinergic nerve cells]. Zhongguo xiu fu chong jian wai 
ke za zhi = Chinese journal of reparative and reconstructive surgery. 2012;26(2):158-65.

12. Zhao HB, Ma H, Ha XQ, Zheng P, Li XY, Zhang M, et al. Salidroside induces rat mesenchymal stem cells to differentiate into dopaminergic neurons. Cell Biol Int. 2014;38(4):462-71.

13. Zuo W, Yan F, Zhang B, Hu X, Mei D. Salidroside improves brain ischemic injury by activating $\mathrm{PI} 3 \mathrm{~K} / \mathrm{Akt}$ pathway and reduces complications induced by delayed tPA treatment. Eur J Pharmacol. 2018;830:128-38.

14. Zhang $X$, Lai $W$, Ying $X, X u$ L, Chu K, Brown J, et al. Salidroside Reduces Inflammation and Brain Injury After Permanent Middle Cerebral Artery Occlusion in Rats by Regulating PI3K/PKB/Nrf2/NFKB Signaling Rather than Complement C3 Activity. Inflammation. 2019;42(5):1830-42.

15. Zhong Z, Han J, Zhang J, Xiao Q, Hu J, Chen L. Pharmacological activities, mechanisms of action, and safety of salidroside in the central nervous system. Drug Des Devel Ther. 2018;12:1479-89.

16. Ge T, Yu Q, Liu W, Cong L, Liu L, Wang Y, et al. Characterization of bone marrow-derived mesenchymal stem cells from dimethyloxallyl glycine-preconditioned mice: Evaluation of the feasibility of dimethyloxallyl glycine as a mobilization agent. Mol Med Rep. 2016;13(4):3498-506.

17. Jiang J, Dai J, Cui H. Vitexin reverses the autophagy dysfunction to attenuate MCAO-induced cerebral ischemic stroke via mTOR/Ulk1 pathway. Biomed Pharmacother. 2018;99:583-90.

18. Pin-Barre C, Laurin J, Felix MS, Pertici V, Kober F, Marqueste T, et al. Acute neuromuscular adaptation at the spinal level following middle cerebral artery occlusion-reperfusion in the rat. PLoS one. 2014;9(2):e89953.

19. Goldman SA. Stem and Progenitor Cell-Based Therapy of the Central Nervous System: Hopes, Hype, and Wishful Thinking. Cell stem cell. 2016;18(2):174-88.

20. Toma C, Pittenger MF, Cahill KS, Byrne BJ, Kessler PD. Human mesenchymal stem cells differentiate to a cardiomyocyte phenotype in the adult murine heart. Circulation. 2002;105(1):93-8.

21. Li Q, Guo Y, Chen F, Liu J, Jin P. Stromal cell-derived factor-1 promotes human adipose tissue-derived stem cell survival and chronic wound healing.Exp Ther Med. 2016;12(1):45-50.

22. Huang L, Zhang L. Neural stem cell therapies and hypoxic-ischemic brain injury. Prog Neurobiol. 2019;173:1-17.

23. Qin YJ, Zeng YS, Zhou CC, Li Y, Zhong ZQ. [Effects of Rhodiola rosea on level of 5hydroxytryptamine, cell proliferation and differentiation, and number of neuron in cerebral hippocampus of rats with depression induced by chronic mild stress]. Zhongguo Zhong yao za zhi = China journal of Chinese materia medica. 2008;33(23):2842-6.

24. Díaz Lanza AM, Abad Martínez MJ, Fernández Matellano L, Recuero Carretero C, Villaescusa Castillo L, Silván Sen AM, et al. Lignan and phenylpropanoid glycosides from Phillyrea latifolia and their in vitro anti-inflammatory activity. Planta medica. 2001;67(3):219-23.

25. Ju L, Wen X, Wang C, Wei Y, Peng Y, Ding Y, et al. Salidroside, A Natural Antioxidant, Improves $\beta$-Cell Survival and Function via Activating AMPK Pathway. Front Pharmacol. 2017;8:749. 
26. Palmeri A, Mammana L, Tropea MR, Gulisano W, Puzzo D. Salidroside, a Bioactive Compound of Rhodiola Rosea, Ameliorates Memory and Emotional Behavior in Adult Mice. J Alzheimers Dis. 2016;52(1):65-75.

27. Chen T, Ma Z, Zhu L, Jiang W, Wei T, Zhou R, et al. Suppressing Receptor-Interacting Protein 140: a New Sight for Salidroside to Treat Cerebral Ischemia. Mol Neurobiol. 2016;53(9):6240-50.

28. Gao J, He H, Jiang W, Chang X, Zhu L, Luo F, et al. Salidroside ameliorates cognitive impairment in a d-galactose-induced rat model of Alzheimer's disease. Behav Brain Res. 2015;293:27-33.

29. Zhang S, Chen X, Yang Y, Zhou X, Liu J, Ding F. Neuroprotection against cobalt chloride-induced cell apoptosis of primary cultured cortical neurons by salidroside. Mol Cell Biochem. 2011;354(1-2):16170.

30. Tan CB, Gao M, Xu WR, Yang XY, Zhu XM, Du GH. Protective effects of salidroside on endothelial cell apoptosis induced by cobalt chloride. Biol Pharm Bull. 2009;32(8):1359-63.

31. Yan R, Xu H, Fu X. Salidroside protects hypoxia-induced injury by up-regulation of miR-210 in rat neural stem cells. Biomed Pharmacother. 2018;103:1490-7.

32. Gui D, Cui Z, Zhang L, Yu C, Yao D, Xu M, et al. Salidroside attenuates hypoxia-induced pulmonary arterial smooth muscle cell proliferation and apoptosis resistance by upregulating autophagy through the AMPK-mTOR-ULK1 pathway.BMC Pulm Med. 2017;17(1):191.

33. Laroni A, Novi G, Kerlero de Rosbo N, Uccelli A. Towards clinical application of mesenchymal stem cells for treatment of neurological diseases of the central nervous system. J Neuroimmune Pharmacol. 2013;8(5):1062-76.

34. Li F, Zhao SZ. Mesenchymal stem cells: Potential role in corneal wound repair and transplantation. World J Stem Cells. 2014;6(3):296-304.

35. Yun HM, Kim HS, Park KR, Shin JM, Kang AR, il Lee K, et al. Placenta-derived mesenchymal stem cells improve memory dysfunction in an AB1-42-infused mouse model of Alzheimer's disease. Cell Death Dis. 2013;4(12):e958.

36. Ha XQ, Yang B, Hou HJ, Cai XL, Xiong WY, Wei XP. Protective effect of rhodioloside and bone marrow mesenchymal stem cells infected with HIF-1-expressing adenovirus on acute spinal cord injury. Neural Regen Res. 2020;15(4):690-6.

37. Shi TY, Feng SF, Xing JH, Wu YM, Li XQ, Zhang N, et al. Neuroprotective effects of Salidroside and its analogue tyrosol galactoside against focal cerebral ischemia in vivo and $\mathrm{H}_{2} \mathrm{O}_{2}$-induced neurotoxicity in vitro. Neurotox Res. 2012;21(4):358-67.

38. Han T. Effects of salidroside pretreatment on expression of tumor necrosis factor-alpha and permeability of blood brain barrier in rat model of focal cerebralischemia-reperfusion injury. Asian Pac J Trop Med. 2013;6(2):156-8.

\section{Figures}


a

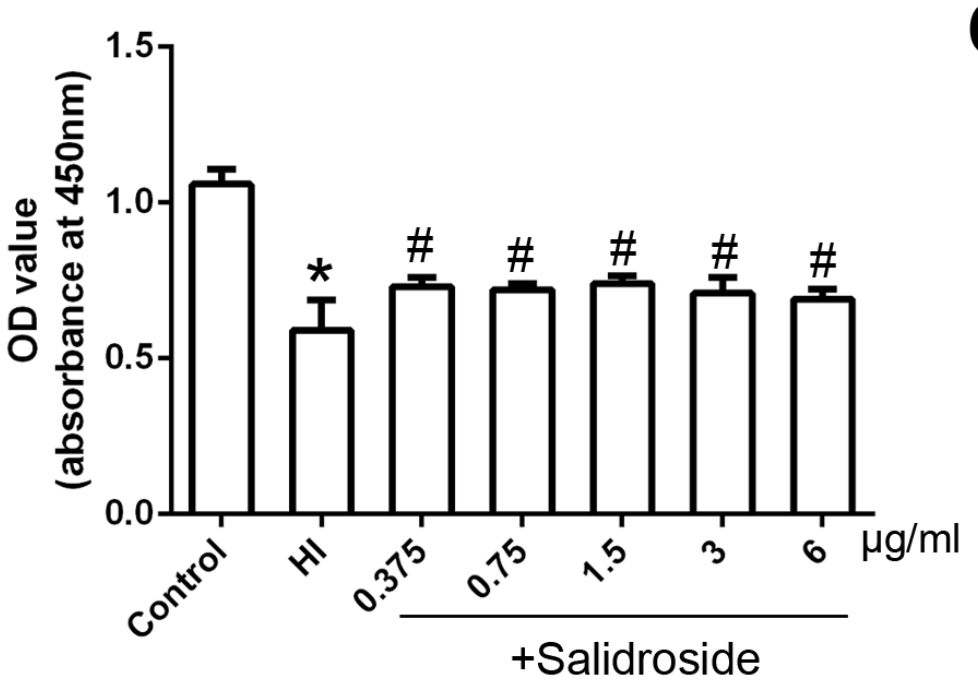

C

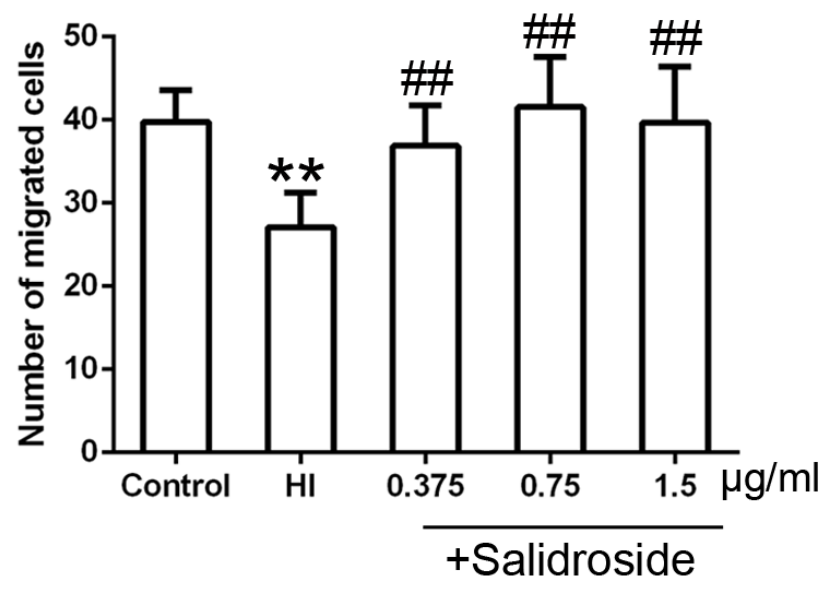

+Salidroside

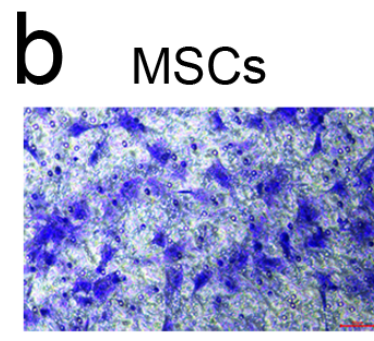
$\mathrm{HI}$ $0.375 \mu \mathrm{g} / \mathrm{ml}$ $0.75 \mu \mathrm{g} / \mathrm{ml}$ $1.5 \mu \mathrm{g} / \mathrm{ml}$
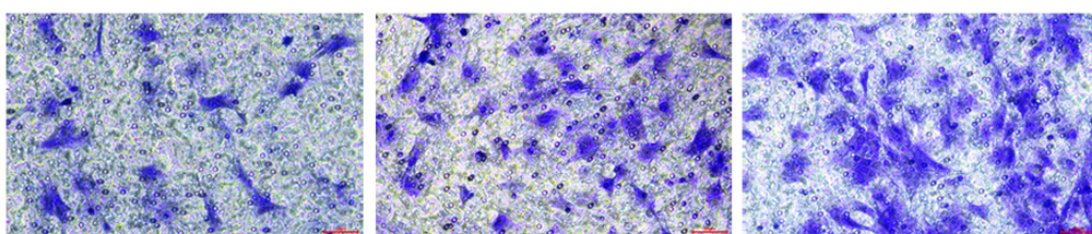

Figure 1

The proliferative ability and migratory capacity of MSCs were promoted by salidroside under hypoxiaischemia. a Cell proliferation was detected by Cell Counting Kit-8. b Cell migration was examined by transwell assay. Migrated cells were stained with crystal violet. c Quantification of migration results. ${ }^{\star} \mathrm{P}<0.05$ and ${ }^{* \star} \mathrm{P}<0.01$ versus control group, $\# \mathrm{P}<0.05$ and $\# \# \mathrm{P}<0.01$ versus vehicle group. 

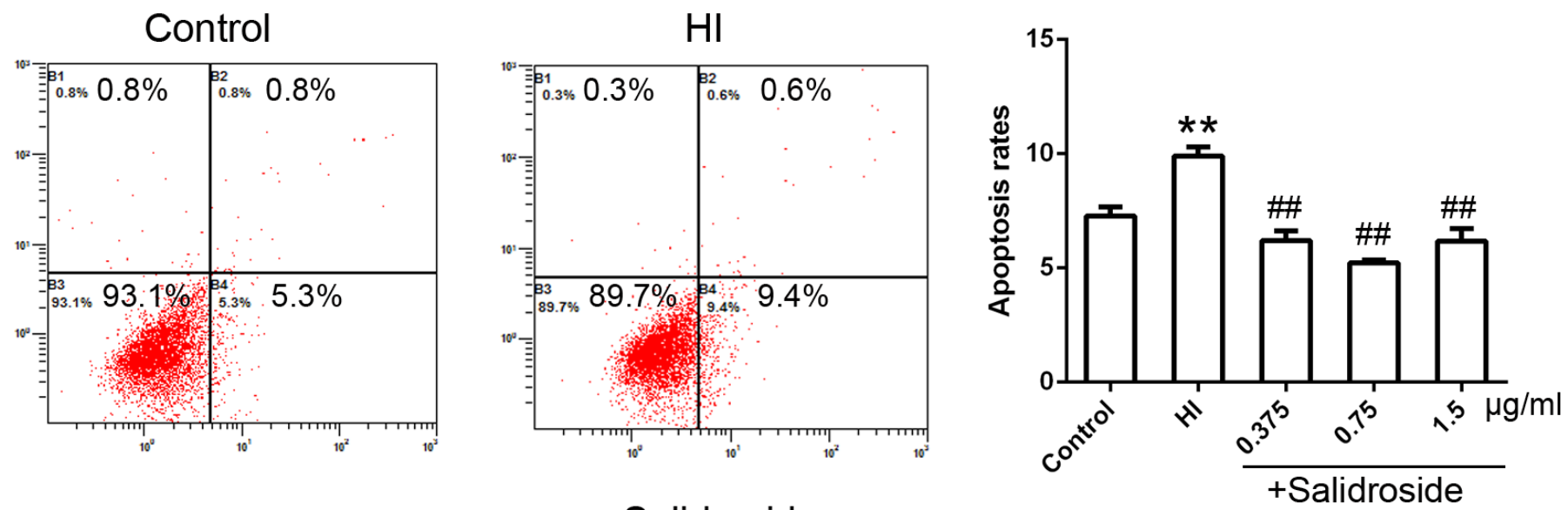

+ Salidroside
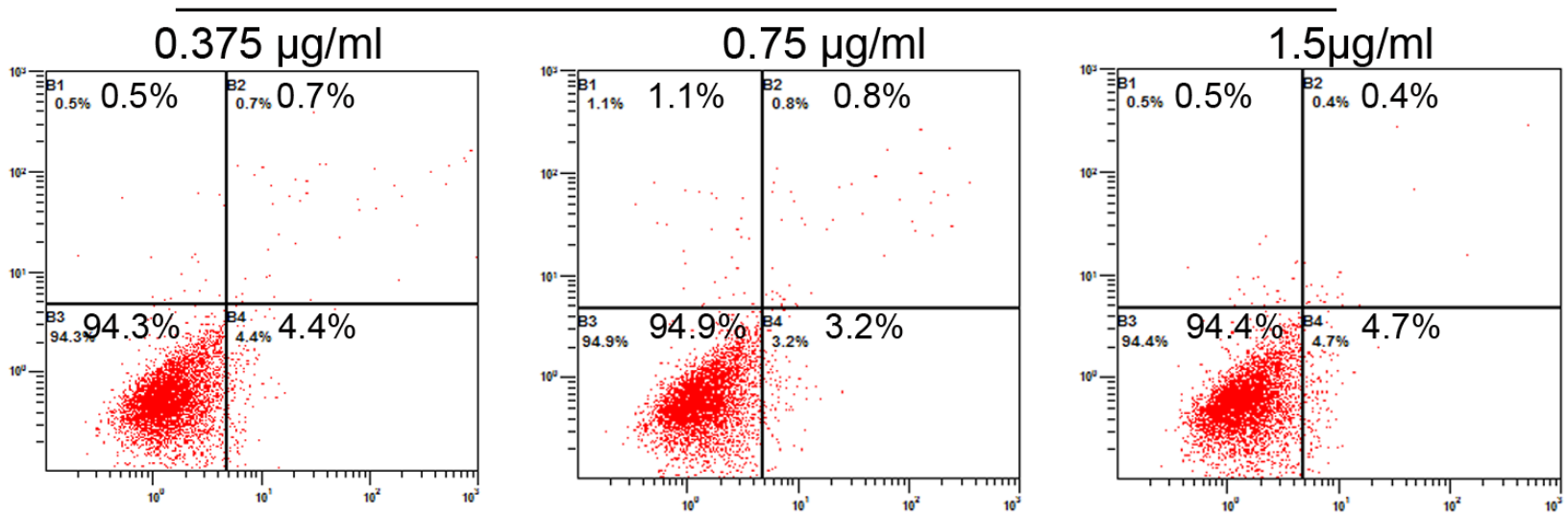

Figure 2

The apoptosis rate of MSCs under hypoxic-ischemic conditions was reduced by salidroside. ${ }^{*} \mathrm{P}<0.01$ versus control group, \#\#P<0.01 versus vehicle group. 

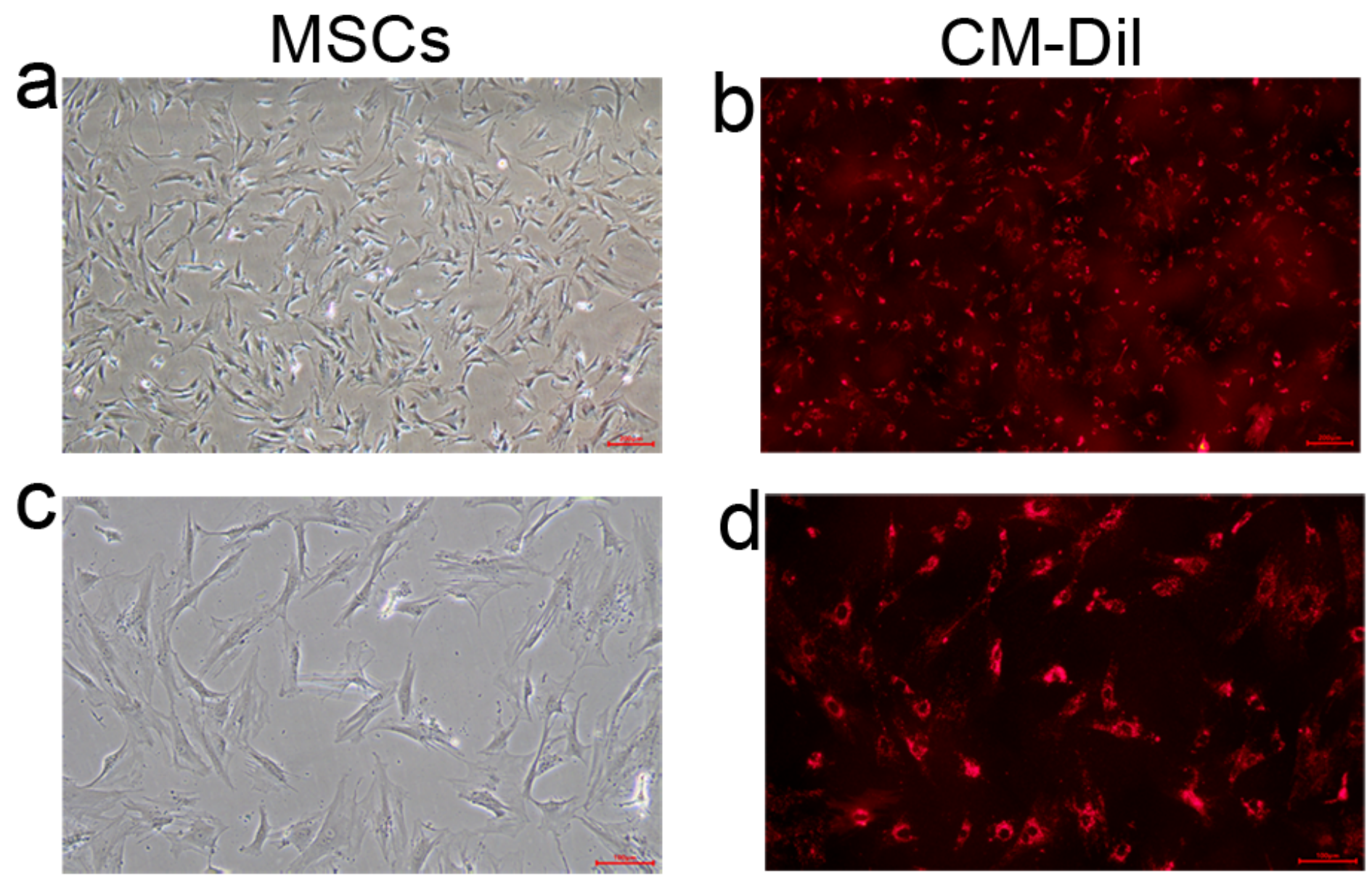

Figure 3

MSCs were labeled with CM-Dil in vitro. a and c MSCs from passage 3 were observed under the inverted fluorescence microscope. $b$ and $d$ The phase-contrast and fluorescent image of CM-Dil labeled MSCs (red). 


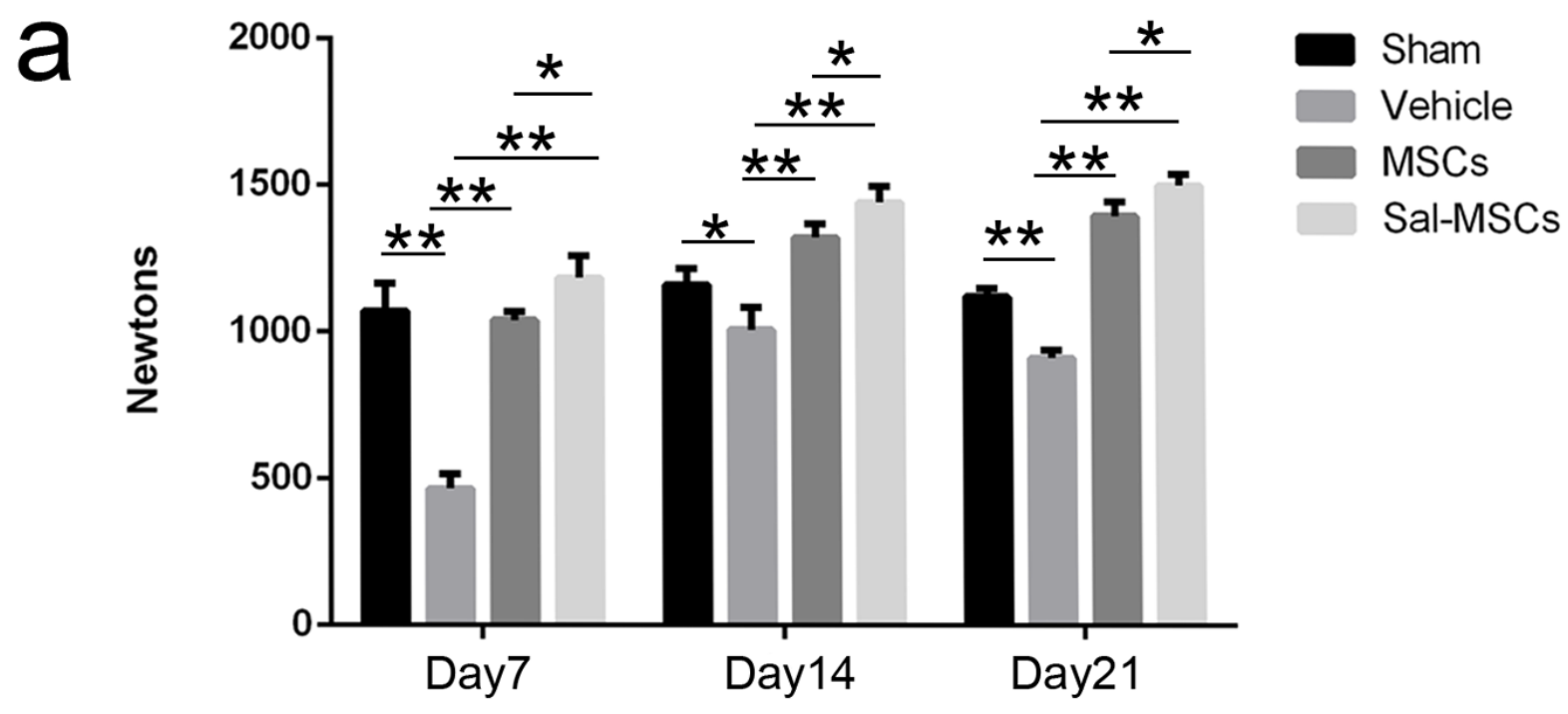

b

Sham Vehicle MSCs Sal-MSCs

C
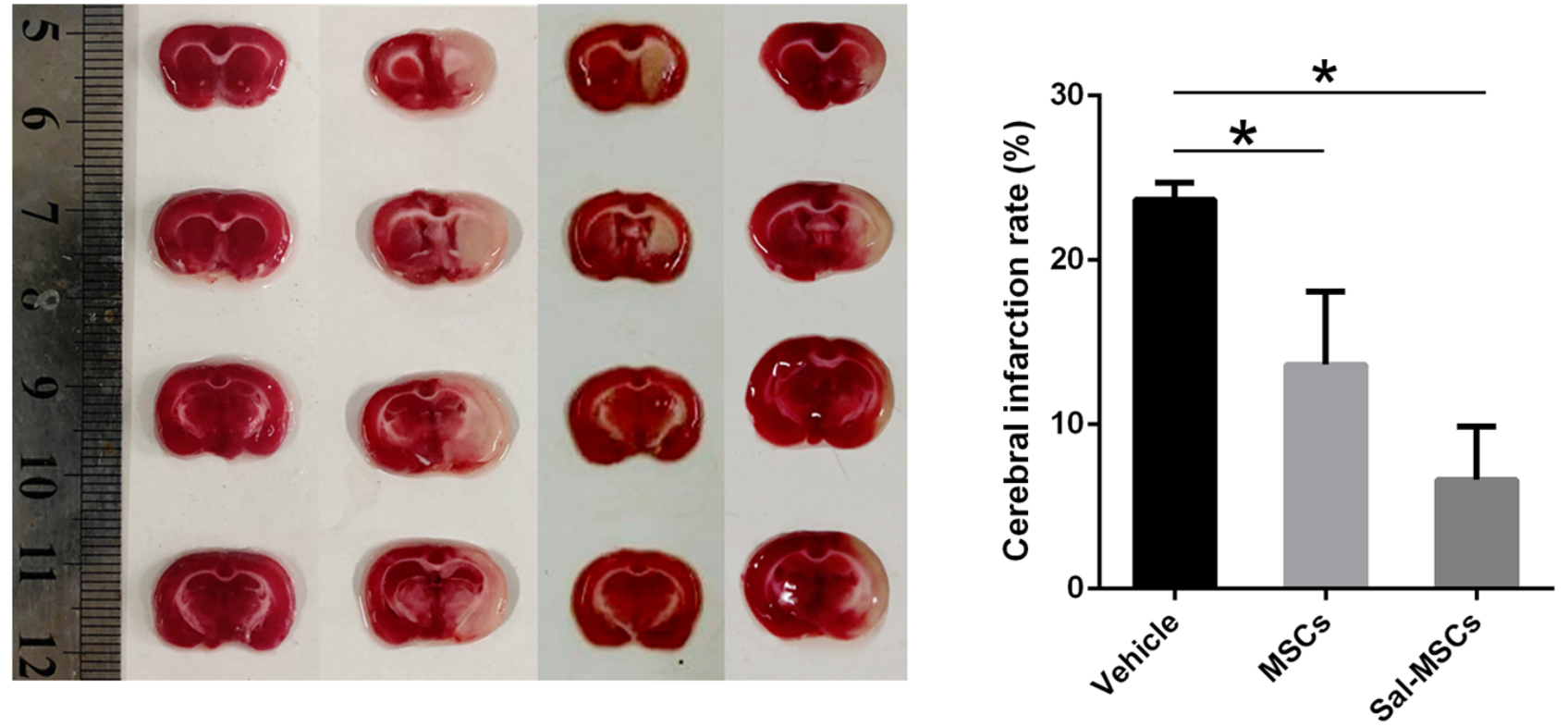

Figure 4

Salidroside-pretreated MSCs ameliorated brain injuries in MCAO rats. a Grip strength for rats in sham group and subject to cerebral ischemic injury. b Brain slices were stained with TTC to visualize lesions. C Quantitative analysis of infarction volume. ${ }^{*} \mathrm{P}<0.05,{ }^{*} \mathrm{P}<0.01$. 

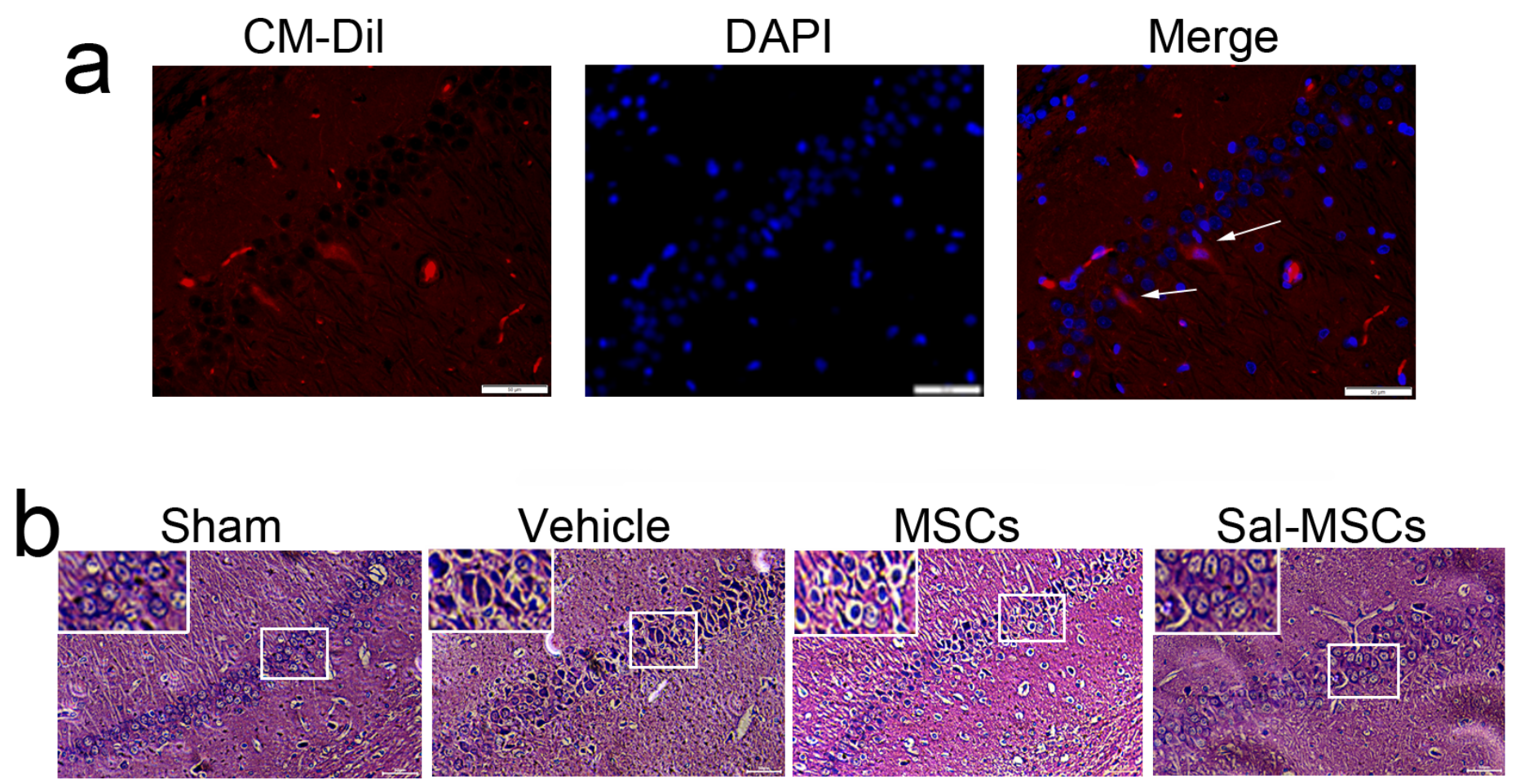

Figure 5

Salidroside-pretreated MSCs alleviated pathological changes in the hippocampal CA1 area after ischemia. a Transplanted MSCs labeled with CM-Dil (red) were observed in the hippocampal CA1 area. b Morphological structures of the hippocampus were evaluated by HE staining.
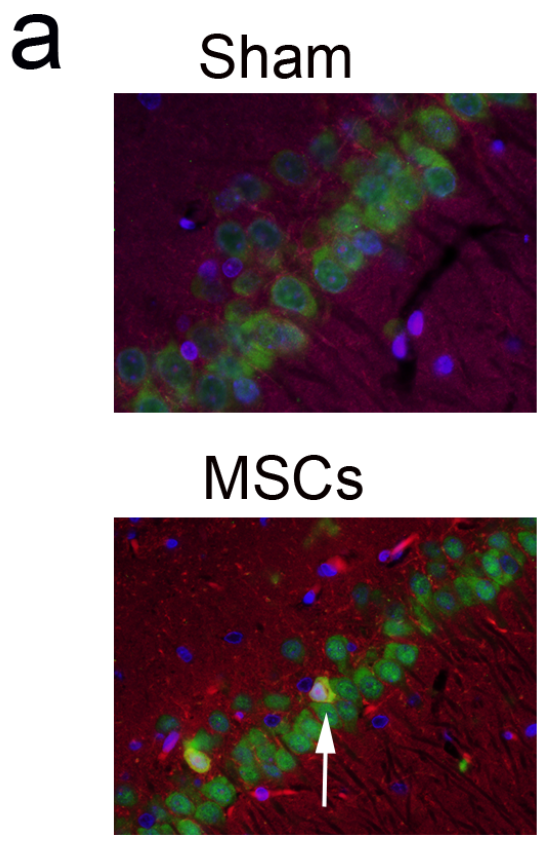
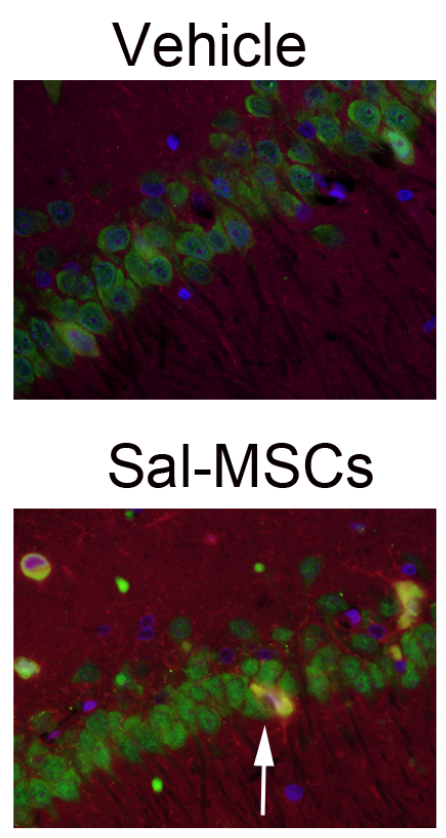

b

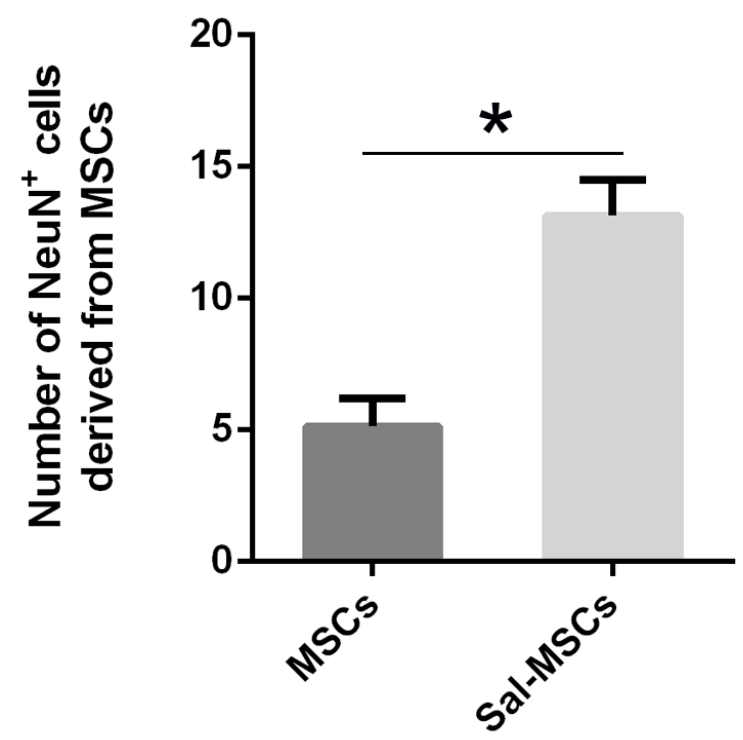

Figure 6 
Salidroside-pretreated MSCs increased the number of NeuN-positive cells in the ischemic hippocampus. a CM-Dil positive cells (red) co-labeled with NeuN (green) were observed in the ischemic boundary zone with yellow fluorescence. Nuclei were counterstained with DAPI (blue). b Quantitative analysis of CM-Dil co-labeled with NeuN-positive cells. ${ }^{*} \mathrm{P}<0.05$.

a
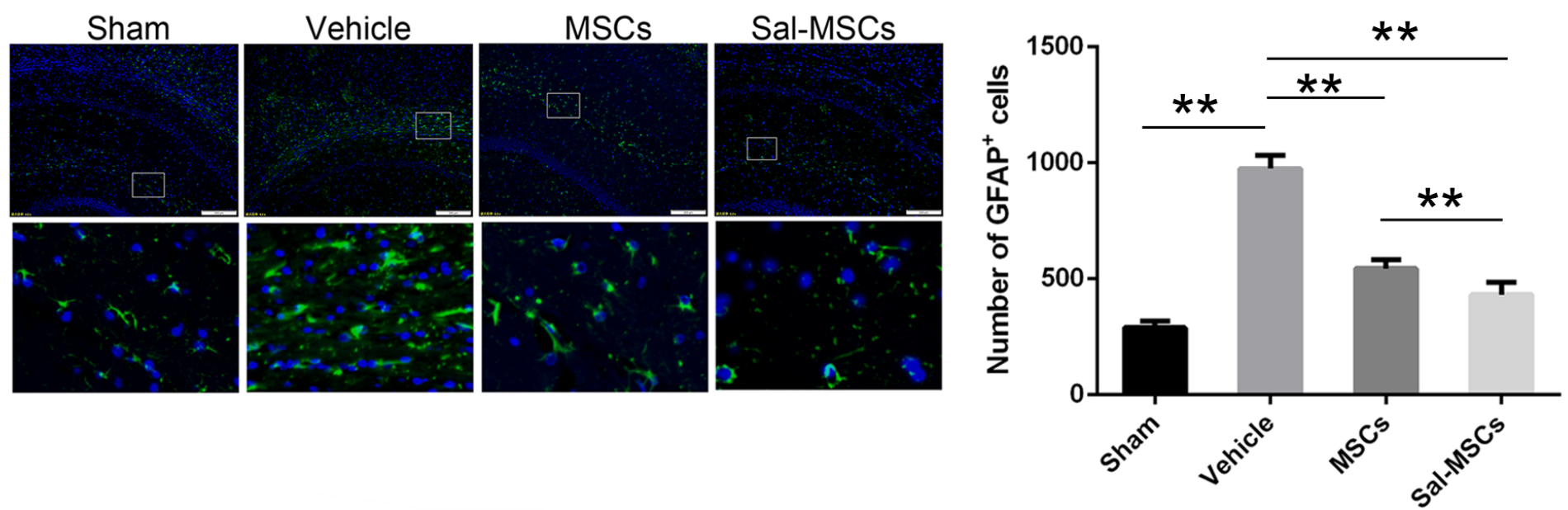

Figure 7

Salidroside-pretreated MSCs decreased the number of GFAP-positive cells in the ischemic hippocampus. a GFAP-positive cells in the ischemic boundary zone with immunolabeled green fluorescence. Nuclei were counterstained with DAPI (blue). b Quantitative analysis of GFAP-positive cells. ${ }^{* \star P}<0.01$. 